\title{
A Bioinspired, Durable, and Nondisposable Transparent Graphene Skin Electrode for Electrophysiological Signal Detection
}

Jiakang Qiu ${ }^{\dagger, \#, \square}$, Tianhao Yu ${ }^{\dagger, \square}$, Weifeng Zhang ${ }^{\dagger}$, Zihan Zhao ${ }^{\dagger}$, Yan Zhang ${ }^{\dagger}$, Guo Ye ${ }^{\dagger}$, Yan Zhao ${ }^{\dagger}$, Xiaojia Du ${ }^{\dagger}$, Xu Liu ${ }^{*}$ Lu Yang ${ }^{*}$ Lijuan Zhang, Shuyan Qi ${ }^{\dagger}$, Qishuo Tan ${ }^{\dagger}$, Xinyu Guo ${ }^{\dagger}$, Guanmeng Li $i^{\dagger}$, Shaoshi Guo ${ }^{\dagger}$, Huiyuan Sun ${ }^{\#}$, Di Wei ${ }^{\ddagger}$, Nan Liu ${ }^{*}, \dagger$,

$\dagger$ Beijing Key Laboratory of Energy Conversion and Storage Materials, College of Chemistry, Beijing Normal University, Beijing 100875, People's Republic of China

$\$$ Beijing Graphene Institute, Beijing 100094, People’s Republic of China

\#College of Physics Science \& Information Engineering and Hebei Advanced Thin

Film Laboratory, Hebei Normal University, Shijiazhuang, Hebei 050024, People's

Republic China

J. Qiu and T. Yu contributed equally to this work.

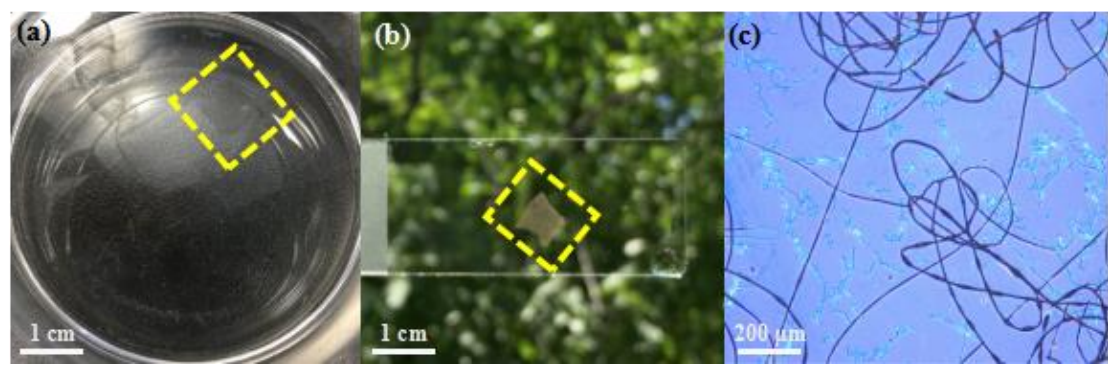

Figure S1. (a-c) Representative optical images showing the fibers on the CVD-grown graphene by electrospinning for $200 \mu \mathrm{L}$.

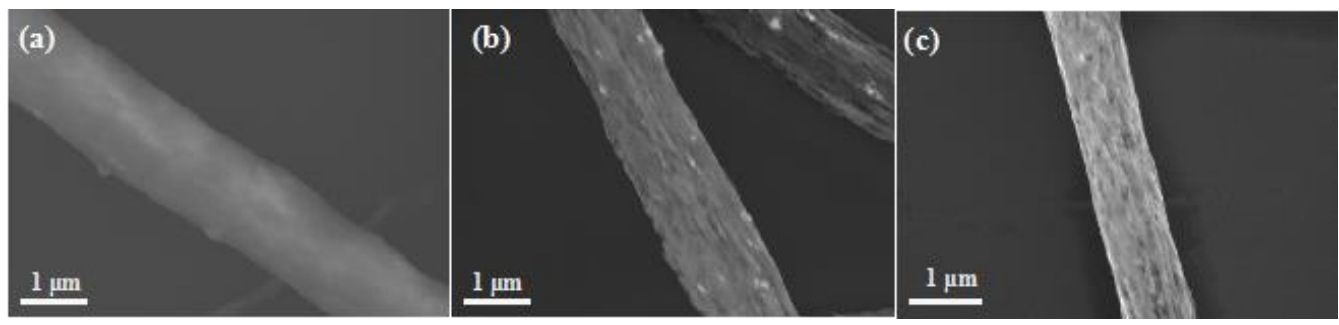

Figure S2. SEM image of fibers/Graphene on the $\mathrm{SiO}_{2} / \mathrm{Si}$ substrate (a) without annealing (b, c) under $600^{\circ} \mathrm{C}, 1000^{\circ} \mathrm{C}$ respectively. 

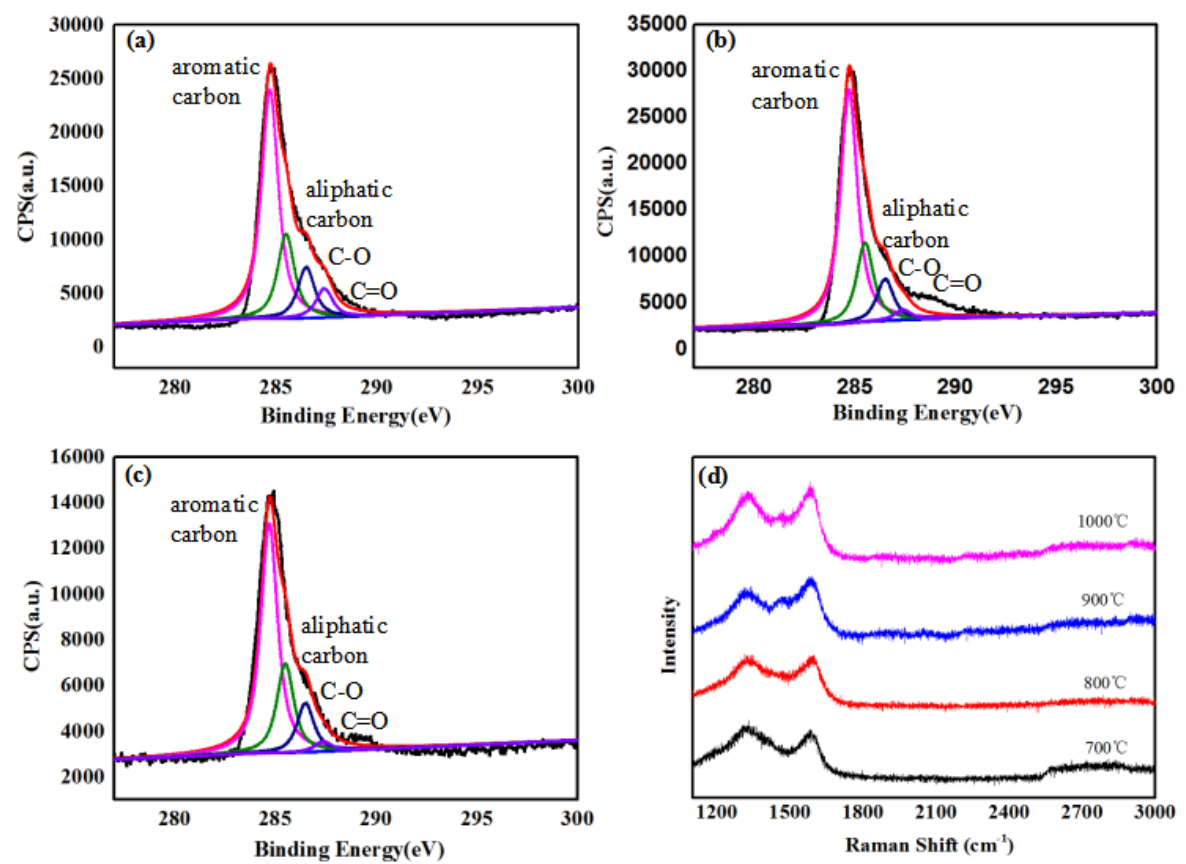

Figure S3. X-ray photoelectron spectrum and Raman spectra. (a) XPS spectrum of annealed fibers/Graphene film without annealing. (b, c) XPS spectrum of annealed fibers/Graphene film under $600^{\circ} \mathrm{C}, 1000^{\circ} \mathrm{C}$ respectively. Peaks at $284.7 \mathrm{eV}, 285.5 \mathrm{eV}, 286.5 \mathrm{eV}$ and $287.4 \mathrm{eV}$ represent hydrocarbon, aliphatic carbon, hydroxyl and carbonyl carbon $\mathrm{C}$ respectively. (d) Raman spectra of annealed fibers/Graphene film annealed at different temperatures.

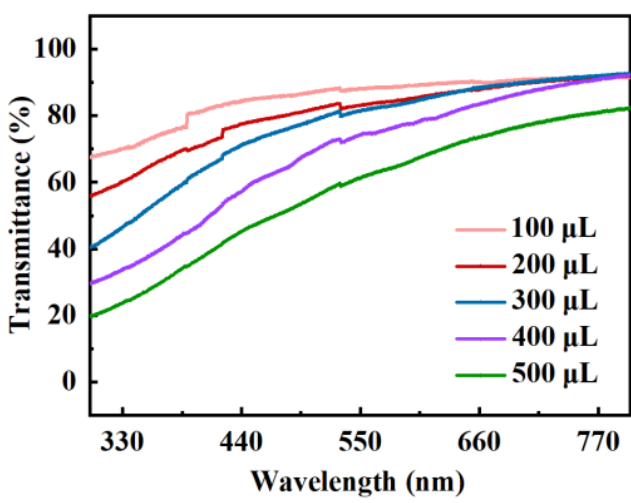

Figure S4. Transmittance spectra for different solution volume. 


\section{Without fibers}

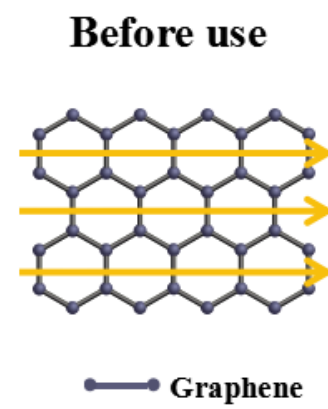

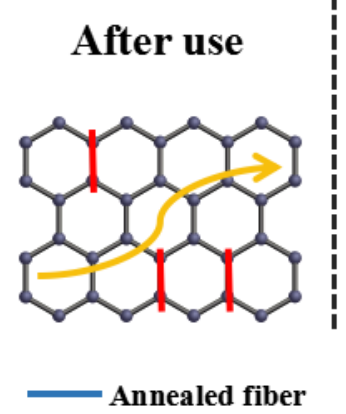

With fibers

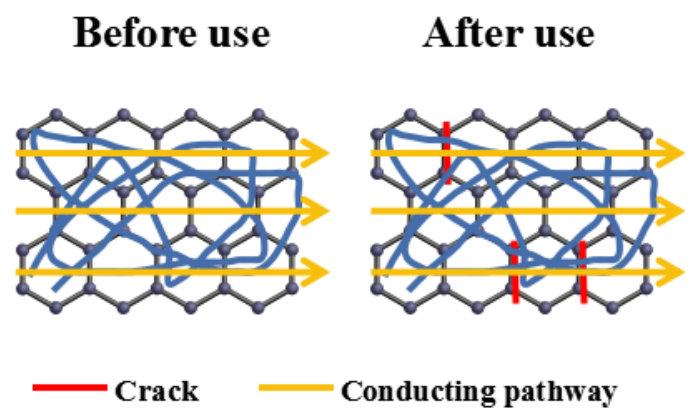

Figure S5. Schematic diagram of GFG and G showing the effects of more conductive channels.

\section{Washing and usage on the durability of semi-embedded GFG in SEBS:}

Washing brings about the reduction of graphene, and it would cause mechanical damage as well. Mechanical damage would occur due to surface tension when the skin-electrodes get rid of water. On the other hand, usage of skin-electrode involves attaching-detaching from skin, which is mainly a type of peeling force. In the still sEMG test, the skin will be weakly deformed, and most of them will be within $3 \%$. It can be seen that the deformation is not enough to cause major damage. Thus, both washing and usage would mainly result in reduction of conductive channels. There are two ways of our electrode to conquer the reduction of conductive channels. One is the semi-embedded structure, and the other is the soldering of electrospun fibers and graphene by high-temperature annealing. Below we compared normalized SNR changes after washing and using respectively (Figure S6). They two demonstrated similar falling trends, indicating both washing and usage would take negative impacts on the electrodes. However, compared with pure graphene, our electrode patch is still less interfered to the damage of washing and using. To further enhance the durability of GFG, dense graphitized electrospun fibers soldering on multi-layer graphene are required to be semi-embedded in SEBS. 


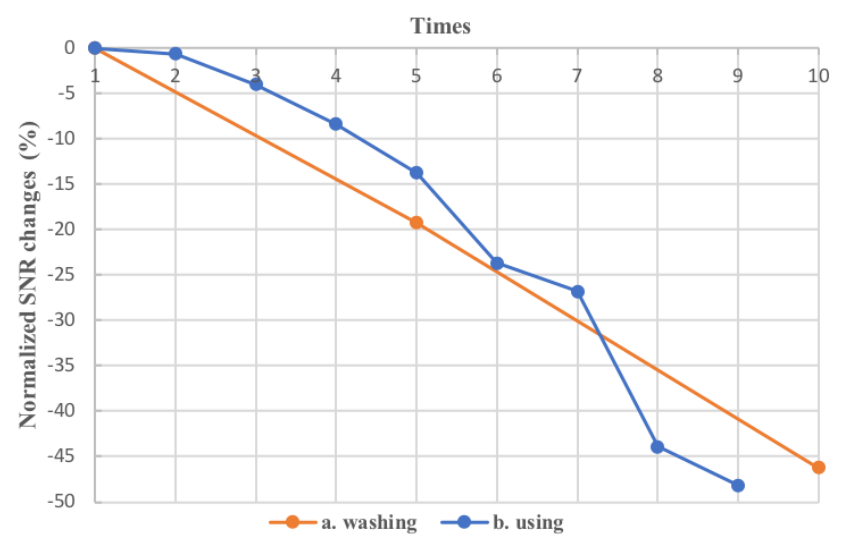

Figure S6. Normalized SNR changes after washing (orange) and using (blue) respectively.

1

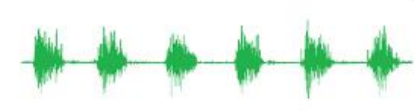

4

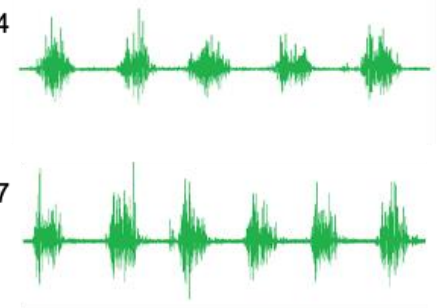

2

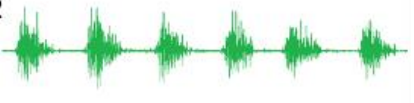

5

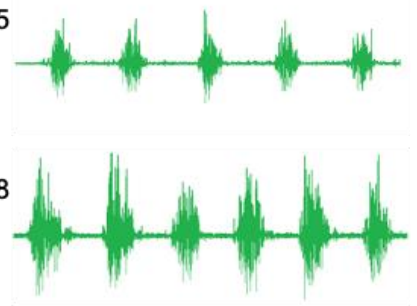

3

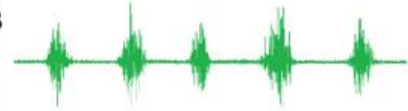

6

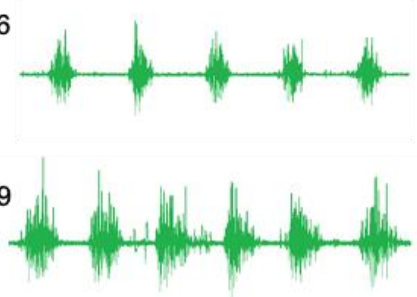

Figure S7. Representative sEMG tests showing 9 repetitive uses of GFG semi-embedded in SEBS.

\section{Safety issue of GFG-SEBS electrode regarding $\mathrm{Cu}^{2+}$ :}

There are two possible forms of $\mathrm{Cu}^{2+}$ in semi-embedded GFG in SEBS, which are free form on the surface and confined form in graphitized electrospun fiber. 1). For the $\mathrm{Cu}^{2+}$ on the surface: the as-prepared electrode has been dealt with deionized water before use. Free form of $\mathrm{Cu}^{2+}$ on the surface could be washed out as much as possible. 2). For the $\mathrm{Cu}^{2+}$ confined in graphitized electrospun fiber: it is inside the graphitized fiber, and since the graphene is contacting the skin as the conductive layer, it would prevent the $\mathrm{Cu}^{2+}$ from penetrating. Overall, safety issue of this skin-electrode regarding $\mathrm{Cu}^{2+}$ can be ignored by carefully washing in deionized water before use.

To measure the biocompatibility of the GFG electrode, we performed a half-day patch test on a 
volunteer's forearm using conventional $\mathrm{Ag} / \mathrm{AgCl}$ gel-based electrode as the control group (Figure S8). The attachment of the GFG electrode to skin showed no adverse effects, including erythema or complications, at the attached site. However, the conventional $\mathrm{Ag} / \mathrm{AgCl}$ gel-based electrodes showed erythema at the attached site, which is suspected to be an allergic reaction. The biocompatibility test results suggest that the GFG electrode is compatible with skin and would be suitable for a relatively long-term monitoring.

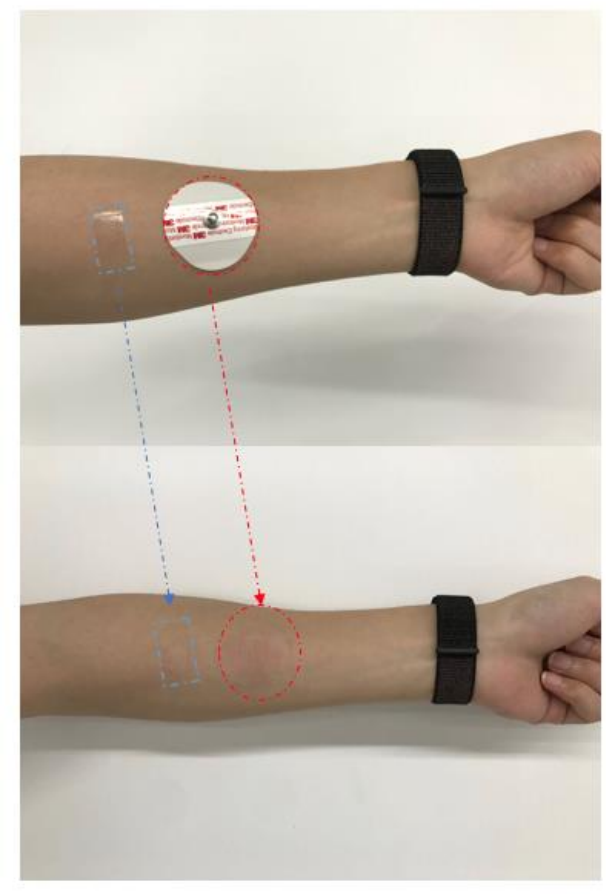

Figure S8. The biocompatibility test of the GFG electrode, using conventional $\mathrm{Ag} / \mathrm{AgCl}$ gel-based electrode as the control group.
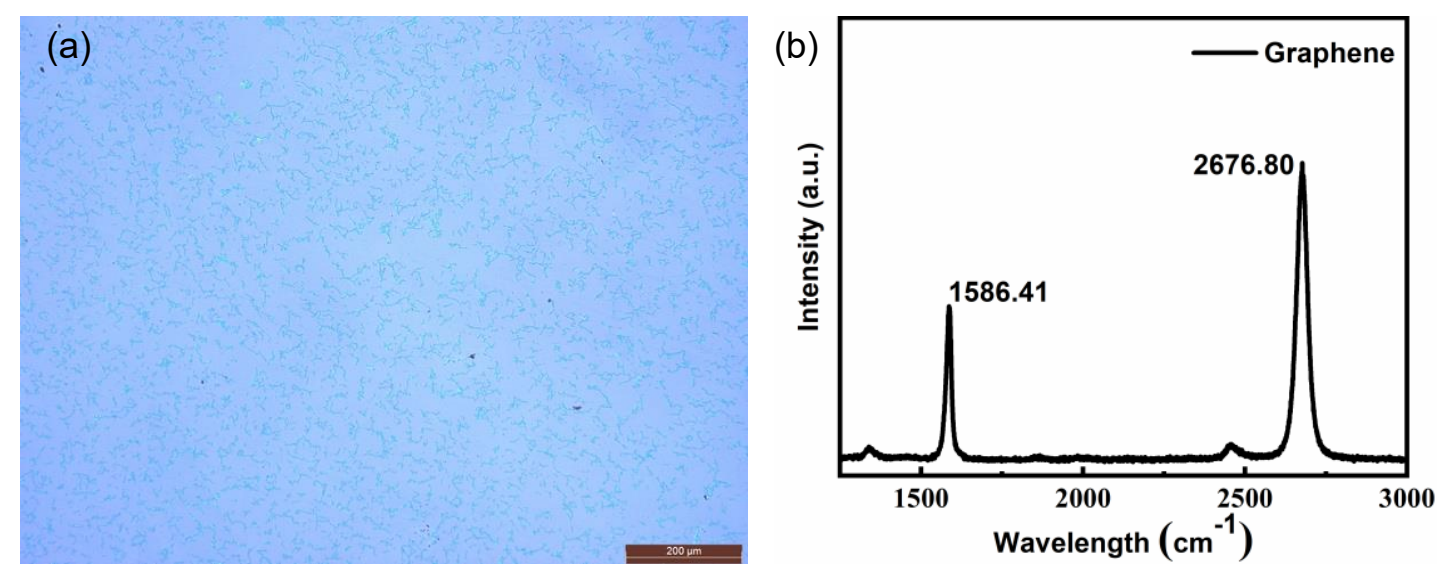

Figure S9. (a) Optical microscope image of the monolayer graphene on 300nm-SiO2/Si substrate. (b) Raman spectrum of the monolayer graphene on $300 \mathrm{~nm}-\mathrm{SiO}_{2} / \mathrm{Si}$ substrate. 
Table S1. Comparison of figure of merits.

\begin{tabular}{|l|l|l|}
\hline Materials & Main Features & Reference \\
\hline Au film & 30.8dB while detecting sEMG & 1 \\
\hline Au nanomesh & gas-permeable & 2 \\
\hline Au/PET & wireless communication & 3 \\
\hline Ag-Au & implantable & 4 \\
\hline $\begin{array}{l}\text { graphitized } \\
\text { electrospun } \\
\text { fiber/SEBS/graphene }\end{array}$ & $\begin{array}{l}\text { 83\% transmittance, } \sim 150 \Omega / \square \text { and up to 30dB while } \\
\text { detecting sEMG, reusable }\end{array}$ & This work \\
\hline
\end{tabular}

\section{References}

1. Nawrocki, R. A.; Jin, H.; Lee, S.; Yokota, T.; Sekino, M.; Someya, T. Self - Adhesive and Ultra Conformable, Sub - 300 nm Dry Thin - Film Electrodes for Surface Monitoring of Biopotentials. Adv. Funct. Mater. 2018, 28, 1803279.

2. Miyamoto, A.; Lee, S.; Cooray, N. F.; Lee, S.; Mori, M.; Matsuhisa, N.; Jin, H.; Yoda, L.; Yokota, T.; Itoh, A. Inflammation-free, gas-permeable, lightweight, stretchable on-skin electronics with nanomeshes. Nat. Nanotechnol. 2017, 12, 907.

3. Yang, S.; Chen, Y. C.; Nicolini, L.; Pasupathy, P.; Sacks, J.; Su, B.; Yang, R.; Sanchez, D.; Chang, Y. F.; Wang, P.; Schnyer, D.; Neikirk, D.; Lu, N. "Cut-and-Paste" Manufacture of Multiparametric Epidermal Sensor Systems. Adv. Mater. 2015, 27, 6423-30.

4. Choi, S.; Han, S. I.; Jung, D.; Hwang, H. J.; Lim, C.; Bae, S.; Park, O. K.; Tschabrunn, C. M.; Lee, M.; Bae, S. Y.; Yu, J. W.; Ryu, J. H.; Lee, S.-W.; Park, K.; Kang, P. M.; Lee, W. B.; Nezafat, R.; Hyeon, T.; Kim, D.-H. Highly conductive, stretchable and biocompatible Ag-Au core-sheath nanowire composite for wearable and implantable bioelectronics. Nat. Nanotechnol. 2018, 13, 1048-1056. 